\title{
Computation of Incipient Separation Via Solution of the Vorticity Equation on a Lagrangian Mesh
}

\author{
Stephen A. Huyer and John R. Grant \\ Naval Undersea Warfare Center \\ Hydrodynamics Branch \\ Newport, RI 02841, USA
}

\begin{abstract}
A novel vorticity based solution methodology has been developed to compute unsteady flow, particularly separation. The vorticity of the flow is determined on a set of points and the vorticity in the field is computed by linear interpolation. This is accomplished by the construction of a connected set of triangular elements formed by Delaunay triangularization of the points in the field. Triangulation of the vorticity field enables computation of first and second order spatial derivatives of the vorticity field using a least squares formulation. An effective diffusion transport velocity was developed to account for spatial movement of the vorticity due to viscosity. Diffusion velocity and direct calculation of the Laplacian allows for a deterministic solution of viscous diffusion. The points are then advected at both the induced velocity (computed from the Biot-Savart integral) and the diffusion velocity. This solution scheme was found to be stable as it was applied to the problem of impulsively started flow about a NACA 0012 airfoil. The phenomena of incipient separation as well as initial development of the unsteady wake was examined.
\end{abstract}

\section{Introduction}

Many current research efforts demonstrate the effectiveness of vortex methods to accurately compute complex unsteady flow fields. The velocity-vorticity formulation of the Navier Stokes equations is used to solve for these flow variables on a Lagrangian mesh. This solution methodology has several advantages over traditional finite difference methods. The method is grid free, is naturally adaptive to coherent vortex structures and is subject to little numerical diffusion. The use of a Lagrangian mesh allows for the straightforward treatment of moving surfaces and does not require incorporation of additional terms due to non-inertial reference frames. Some applications of vortex methods to engineering problems of interest have demonstrated the potential to solve complex high Reynolds number unsteady flow fields.

Traditional vortex methods which utilize point vortices or vortex blobs have certain limitations which make them difficult to apply to engineering problems. One important limitation is the isotropy of the geometry of the blob, which requires many blobs to properly resolve a highly anisotropic flow such as a boundary layer. We have developed a technique using interpolation by triangularization of the vorticity field. This method has the advantage that the elements are connected so it is possible to compute derivatives and therefore deterministically solve for viscous diffusion as well as to directly calculate the Biot-Savart integral. This approach is similar to the methods documented by Russo and Strain [8] who examined inviscid vorticity fields in the absence of surfaces. In our method, the vorticity is specified on a number of points in the field. These 
points are then connected to form a set of triangles via a Delaunay triangularization algorithm (Peraire et al. [7], also described by Russo and Strain [8]). The vorticity at any other point in the region is then approximated by linear interpolation based on the vorticity at the nodal points and shape functions specified by the geometry of the triangle. Since the vorticity is linear with respect to space, the spatial derivatives over the triangle are constant. First and second order spatial derivatives are approximated to higher order via a second order least squares formulation to include all the triangles intersecting a given node. The vorticity field and the locations of the calculation points are updated each time step. Since a Lagrangian formulation is used, the advection term is automatically included. Viscous diffusion is accomplished using both an effective diffusion velocity (i.e. Kempka and Strickland [5]) as well as the second order Laplacian. Flow field velocities were computed via direct integration of the Biot-Savart integral. A generic fast computation algorithm which could be readily extended to 3D problems was used for both the Delaunay triangularization as well as computation of the velocity field.

To demonstrate the effectiveness of the present method, flow past an airfoil at angle of attack was examined. The flow was rapidly accelerated from 0.0 to 1.0 to effect an impulsive start. Velocities are non-dimensionalized using the freestream velocity and distances are non-dimensionalized using the chord. The boundary layer and wake were then allowed to develop. The zero angle of attack test case demonstrated attached flow and a smooth boundary layer profile. Angle of attack was then increased beyond stall to examine the formation of the separated region. Incipient and subsequent development of the separated flow region over the airfoil was then examined using surface vorticity and pressure data as well as boundary layer velocity profiles.

\section{Methods}

\subsection{Surface grid}

A representation of a NACA 0012 airfoil was constructed by connecting 200 line segments from body coordinates. This is typical of the way bodies are defined for flow computations by the code. The line segments form the base of the triangles used in the vorticity element calculations. Initially, four layers of nodes are constructed above the surface. Figure 1 shows a section of the surface with this initial nodal vorticity distribution. The distance above the surface of each layer of nodes is based on a diffusion length scale, $l_{d}=\sqrt{4 \nu \Delta t}$. Each layer is separated by a distance of $0.5 l_{d}$ and contain the same number of points used to represent the surface. The source panels are represented by circular arcs and remain non-singular and will be described in the next section.

\subsection{Satisfaction of the velocity boundary conditions}

Each node on the body surface effectively carries two velocity generators: a surface vorticity and a potential source distribution. As will be discussed later, the vorticity amplitudes are specified by the surface boundary conditions at the nodal points on the surface and allowed to diffuse to nodal points in the field. The sources are needed, mathematically, to prevent penetration of the nodal points into the surface and to ensure that both a zero streamline on the body surface and the no-flux boundary conditions are satisfied. Uhlman and Grant [9] showed that as the number of points representing the surface vorticity increases, the strengths of the sources approach zero. The need for the sources is therefore a result of the finite discretization of the surface vorticity distribution.

Non-singular source panels were developed in order to compute the source velocities at the nodal points. The source panel geometry is shown in Fig. 2. For ease of development, polar 
coordinates $(r, \theta)$ were chosen with radial and tangential velocities computed at the $n$th surface nodal coordinate. Three points, $n-1, n$ and $n+1$, are required to determine the radius of a circle and the circle centroid, $x_{c}$ and $y_{c}$. We now desire the velocity at some field point, $x, y$. This is determined by using the integral:

$$
I(\mathbf{x})=\int_{C} f(s) \frac{\left(\mathbf{x}-\mathbf{x}^{\prime}(s)\right)}{\left|\mathbf{x}-\mathbf{x}^{\prime}(s)\right|^{2}} d s
$$

where $s$ is the surface coordinate and $f$ is the surface shape function. $f$ is chosen such that:

$$
f\left(s_{n}\right)=1.0 \text { and } \quad f\left(s_{n \pm 1}\right)=0.0
$$

in polar coordinates, the integrals may be evaluated along $\theta$ for:

$$
f(\theta)= \begin{cases}\frac{\sin \left(\theta_{n+1}-\theta\right)}{\sin \left(\theta_{n+1}-\theta_{n}\right)}, & \theta_{n} \leq \theta \leq \theta_{n+1} \\ \frac{\sin \left(\theta-\theta_{n-1}\right)}{\sin \left(\theta_{n}-\theta_{n-1}\right)}, & \theta_{n-1} \leq \theta \leq \theta_{n}\end{cases}
$$

where $\theta$ is defined in the local coordinate system. This local coordinate system is chosen so that $\hat{p}$ lies along $\mathbf{x}-\mathbf{x}_{c}$. So the unit vectors are:

$$
\hat{p}=\frac{\mathbf{x}-\mathbf{x}_{c}}{\left|\mathbf{x}-\mathbf{x}_{c}\right|}, \hat{q}=\hat{z} \times \hat{x}
$$

This then has the advantage that the local normal coordinate of the field point is:

$$
q=0.0
$$

It can then be shown that the integral, expressed in local polar coordinates, is:

$$
\mathbf{I}_{n}=\hat{p} R \int_{\theta_{n-1}}^{\theta_{n+1}} f(\theta) \frac{p-R \cos \theta}{p^{2}+R^{2}-2 R p \cos \theta} d \theta-\hat{q} R^{2} \int_{\theta_{n-1}}^{\theta_{n+1}} f(\theta) \frac{\sin \theta}{p^{2}+R^{2}-2 R p \cos \theta} d \theta
$$

The integral may be transformed back to the global coordinate system so the velocity is:

$$
\mathbf{u}(\mathbf{x})=-\frac{1}{2 \pi} \sum_{n=1}^{N} \sigma_{n} \mathbf{I}(x)_{n}
$$

where $\sigma_{n}$ are the source amplitudes for node $n$ of a body defined by $N$ points.

The velocities produced by the triangular vorticity elements whose nodes intersect at the surface are shown in the next section. There are $N$ equations produced for the $N$ surface vorticity values. For a body defined by $N$ points, there are then $2 N$ unknowns. Requiring the total velocity (that due to the surface source distribution and the field vorticity) to be zero at each surface node produces an equivalent number of equations. However, the integral of vorticity over a bounded volume is zero when the velocity vanishes on the bounding surface (no-slip condition). This property requires that the integral of the nodal vorticity over the body surface be zero. Circulation about the body will be generated depending on the instantaneous vorticity distribution in the boundary layer. 
Similarly, the integral of the surface potential source over the body surface is zero by continuity. These constraints may be written as:

$$
\begin{gathered}
\frac{1}{2} \sum_{n=1}^{N} \sigma_{n} R_{n}\left(\theta_{n+1}-\theta_{n-1}\right)=0 \\
\frac{1}{3} \sum_{n=1}^{N} \Omega_{n} \sum_{m=1}^{M} A_{m}=0
\end{gathered}
$$

where $m$ refers to the set of elements which intersect the surface node, $n, R$ refers to the arc-length of the surface segment, $\Omega$ is the vorticity amplitude and $A$ is the area of the triangular element. The factor of $1 / 2$ for the source summation is effectively due to the average over the two arc lengths inclusive of node $n$. Similarly, the factor of $1 / 3$ for the vorticity element summation is due to the average vorticity from the three nodal vorticity amplitudes for a triangle. This set of $2 N+2$ equations is an over determined set. The matrix solution for the surface strengths is formulated via Lagrange multipliers so that the integral constraints are met exactly and the $2 N$ boundary conditions on velocity are satisfied in a least-squares sense. The deviation from zero of the velocity at the control points has always been found to be slight; maximum values of this deviation for the computations described below are of order $10^{-3}$ of freestream velocity.

\subsection{Linearized triangular vorticity elements}

In the present method, nodal vorticity values are known and a linear variation of vorticity between nodal points is assumed. At each time step, a Delaunay triangularization routine is used to form an unstructured mesh connecting each nodal point thus forming triangular elements. Consider a single triangular element (Fig. 3) with nodes numbered 1, 2 and 3 with vertices located at $\left(x_{1}, y_{1}\right)$, $\left(x_{2}, y_{2}\right)$, and $\left(x_{3}, y_{3}\right)$. Then, in area coordinates (see Zienkiewicz [11]):

$$
\begin{aligned}
& L_{1}=\left(a_{1}+b_{1} x+c_{1} y\right) / 2 \Delta \\
& L_{2}=\left(a_{2}+b_{2} x+c_{2} y\right) / 2 \Delta \\
& L_{3}=\left(a_{3}+b_{3} x+c_{3} y\right) / 2 \Delta
\end{aligned}
$$

where:

$$
\begin{aligned}
\Delta & =\text { triangle area } \\
a_{1} & =x_{2} y_{3}-x_{3} y_{2} \\
b_{1} & =y_{2}-y_{3} \\
c_{1} & =x_{3}-x_{2}
\end{aligned}
$$

similarly for nodes 2 and 3 . Values for the shape functions $\left(L_{1}, L_{2}, L_{3}\right)$ are then 1.0 at their respective nodes and 0 at each of the other two nodes. The vorticity over the element may then be expressed as:

$$
\omega=\Omega_{1} L_{1}+\Omega_{2} L_{2}+\Omega_{3} L_{3}
$$

The velocity (from the Biot-Savart integral) is:

$$
\mathbf{u}(\mathbf{x})=\frac{1}{2 \pi} \int_{A} \boldsymbol{\omega}\left(\mathbf{x}^{\prime}\right) \times \frac{\left(\mathbf{x}-\mathbf{x}^{\prime}\right)}{\left|\mathbf{x}-\mathbf{x}^{\prime}\right|^{2}} d A
$$


Using integration by parts, this integral is split into two parts so:

$$
\mathbf{u}(\mathbf{x})=-\frac{1}{2 \pi}\left(\oint_{c} \boldsymbol{\omega}\left(\mathbf{x}^{\prime}\right) \times \hat{n}(\mathbf{x}) \ln \left|\mathbf{x}-\mathbf{x}^{\prime}\right| d l^{\prime}+\int_{A}\left(\nabla^{\prime} \times \boldsymbol{\omega}\left(\mathbf{x}^{\prime}\right)\right) \ln \left|\mathbf{x}-\mathbf{x}^{\prime}\right| d A\right)
$$

The area integral can be expressed as:

$$
\mathbf{u}_{A}(\mathbf{x})=\left\{\sum_{k=1}^{3} \frac{\left|\Omega_{k}\right|}{4 \pi \Delta}\left[\left(x_{k+1}-x_{k-1}\right) \hat{x}+\left(y_{k+1}-y_{k-1}\right) \hat{y}\right]\right\} E(\mathbf{x}, s)
$$

where

$$
E(\mathbf{x}, s)=\int_{A} \ln \left|\mathbf{x}-\mathbf{x}^{\prime}\right| d A
$$

The circuit integral can be evaluated over each leg of the triangle resulting in an integral of the form:

$$
\mathbf{u}_{c}(\mathbf{x})=\frac{1}{2 \pi} \sum_{k=1}^{3} \hat{n}_{k} \times G(\mathbf{x}, \eta)
$$

where

$$
G(\mathbf{x}, \eta)=\int_{\eta_{k}^{-}}^{\eta_{k}^{+}}\left[\Omega_{k-1} N_{k-1}\left(\mathbf{x}^{\prime}-\mathbf{x}\right)+\Omega_{k} N_{k}\left(\mathbf{x}^{\prime}-\mathbf{x}\right)\right] \ln \left|\mathbf{x}-\mathbf{x}^{\prime}\right| d \eta^{\prime}
$$

where $\eta$ is defined as the segment from the $k-1$ to the $k$ th node.

Since the vorticity is assumed to vary linearly over the element, the first derivatives will be constant over a single element and the second derivatives will be zero. A more accurate way to compute the derivatives was desired. It was found that an accurate way to express first and second order spatial derivatives across scattered points was to construct a least squares solution for all the triangles which intersect a given node. This is accomplished by expressing the vorticity about a desired node as:

$$
\omega=a x+b y+c x^{2}+d y^{2}+e x y
$$

and determining the constants $a, b, c, d$, and $e$ by a least-squares fit to the values of $\omega$ at points in the neighborhood. $x$ and $y$ are referenced to the local node. At a given nodal point $(x=y=0)$ :

$$
\begin{aligned}
\frac{\partial \omega}{\partial x} & =a, \quad \frac{\partial \omega}{\partial y}=b \\
\nabla^{2} \omega & =\frac{\partial^{2} \omega}{\partial x^{2}}+\frac{\partial^{2} \omega}{\partial y^{2}}=2.0(c+d)
\end{aligned}
$$

\subsection{Evolution of the vorticity field}

In inviscid flow, vorticity is transported by the velocity field in the same way as a material element. This type of flow is thus very well suited to Lagrangian mesh formulations, where the mesh points are transported by the velocity field. However, when vorticity is transported by means other than advection by the velocity field, some accounting must be made for this transport in the mesh point distribution. Such is the case for viscous fluids, which transport vorticity via diffusion as well as advection. Rather than introducing new 'empty' points into the mesh onto which vorticity may 
diffuse, we transport the existing mesh points with the sum of a diffusion velocity and the usual flow velocity. Thus the mesh points tend to move from regions of larger vorticity magnitude to regions of lesser magnitude, according to the diffusive transport by viscosity.

The concept of diffusion velocity for scalars (as is the vorticity magnitude, the dependent variable for $2 \mathrm{D}$ flows) is readily developed. Introduce a velocity $\mathbf{v}$ such that the transport $\mathbf{v} \omega$ is the same as the diffusive transport:

$$
\mathbf{v} \omega=-\nu \nabla \omega
$$

Thus for scalars the diffusion velocity is

$$
\mathbf{v}=-\frac{\nu}{\omega} \nabla \omega=-\nu \nabla(\ln \omega)
$$

This is the same form as that developed by Kempka and Strickland [5], although the approach here is different.

Now replace the diffusive flux in the vorticity equation by the product $\mathbf{v} \omega$, yielding

$$
\frac{\partial \omega}{\partial t}+\mathbf{u} \cdot \nabla \omega=-\nabla(\mathbf{v} \omega)
$$

However, the new form here can be manipulated to

$$
\frac{\partial \omega}{\partial t}+(\mathbf{u}+\mathbf{v}) \cdot \nabla(\omega)=\nu \nabla^{2} \omega+\mathbf{v} \cdot \nabla \omega
$$

Thus we have simply introduced the same term on both sides of the vorticity equation. In the process, though, we have identified the proper diffusion velocity to implement into the solution. That is, the mesh points are transported according to the equation

$$
\frac{d \mathbf{x}}{d t}=\mathbf{u}+\mathbf{v}
$$

and the vorticity evolution on these points is specified by (17). We have found that, for numerical purposes, a better-behaved form of (17) is

$$
\frac{\partial(\ln \omega)}{\partial t}+(\mathbf{u}+\mathbf{v}) \cdot \nabla(\ln \omega)=\nu \nabla^{2}(\ln \omega)
$$

although care must be taken where $\omega$ is very small. Also, since the vorticity diffusion term is computed in terms of the natural log function, difficulties arise when the vorticity changes sign. To treat these cases, area weighted averages of the vorticity field surrounding a node is computed. If the vorticity is of opposite sign compared with the node in interest, the vorticity of that node cannot physically increase in magnitude. The Laplacian is then adjusted to ensure that the magnitude of vorticity decreases. Improved methods are currently being examined to address this difficulty.

As the points are continually advected and diffused, the triangles will deform by stretching or compressing. In order to maintain spatial resolution of the vorticity field, a division algorithm was developed. The vorticity at the centroid of the triangle is computed using both the linear interpolation as well as a second order approximation (from the least squares formulation). If the difference in vorticity due to the second order term is $10 \%$ greater than the linear approximation of vorticity, a new nodal point is added at the element centroid. This method ensures that sufficient spatial resolution exists to properly compute second order derivatives. The vorticity at this point is the average of the vorticity amplitudes at each node. To offer additional control on the number 
of points in the field, an amalgamation routine was written as well. If points become closer than $0.1 l_{d}\left(l_{d}\right.$ is the diffusion length scale defined earlier), the nodal vorticity values are combined in such a way as to conserve total circulation. Amalgamation was found to be necessary for the viscous diffusion calculation to remain stable.

In order to increase the resolution of the surface as well as the flow field, it was necessary to compute the interactions of a large set of vorticity elements. The fast multipole method allowed us to compute the flow field due to the increased number vorticity elements in reasonable CPU times. A description of the calculation used in this paper is described by Huyer, Grant and Uhlman [3] and will be not be repeated here. It is based on the method presented by Greengard and Rokhlin [2]. The final expression for each velocity component has the form:

$$
\Psi=\sum_{n=0}^{\infty} \sum_{m=0}^{n} B_{n-m, m} h^{n-m} k^{m}
$$

An expression for the far-field influence of all vorticity elements in terms of a set of coefficients, $B$, and local coordinates relative to the box center, $h, k$, for each box in the field was thus formulated. An examination of the errors due to the fast calculation method were computed. Average and maximum errors were found to be of the order $10^{-4}$ and $10^{-3}$ respectively.

\subsection{Numerical parameters}

Effectiveness of the present formulation was determined by solving the unsteady flow development about a NACA 0012 airfoil. Reynolds number was set to 5000 based on the airfoil chord. Airfoil angle of attack $(\alpha)$ was set at $0^{\circ}, 5^{\circ}, 10^{\circ}, 15^{\circ}$ and $20^{\circ}$. Dimensionless values for airfoil chord and maximum freestream velocity were 1.0. Time was non-dimensionalized by freestream velocity and chord so in one time unit, the freestream moves one chord length. Solutions are presented for linear freestream acceleration from 0.0 at $t=0.0$ to 1.0 at $t=0.1$. Initially, 200 surface nodal vorticity values and four layers of 200 nodes were present at the first time step for a total of 1000 nodes. Time was incremented using a constant time step $(\Delta t)$ of 0.02 . Test runs were carried out to a time of 8.0 where, depending on angle of attack, 8000-10 000 nodes were used to compute the unsteady flow.

All computations were performed on the Cray C-90 at the Naval Oceanographic Office at Stennis Space Flight Center, Mississippi.

\section{Results}

Figure 4 shows the triangular mesh over a NACA 0012 airfoil at $15^{\circ}$ angle of attack at times $t=1.0$ and 7.0. The triangular mesh is shown upstream of $x / c=1.25$ only. Notice how the mesh adapts to the local flow. Increased nodal density is seen in regions where high vorticity gradients are expected with lesser densities in regions of little vorticity. In addition, as the flow evolves, the mesh automatically adapts to changes in the local flow. Nodes are advected thus following individual vortex structures. Consequently, these vortices retain their cohesiveness and are subject to little numerical diffusion.

Figure 5 shows the flow and wake over the NACA 0012 airfoil at $0^{\circ}$ and $5^{\circ}$ angle of attack. A relatively low scale for the vorticity is shown to illuminate the wakes structures. Here, the color plots show the opposite signed vorticity with red counter-clockwise (positive) vorticity and blue clockwise (negative) vorticity. Increased boundary layer thickness can be seen over the airfoil from leading to trailing edge. At the trailing edge, opposite signed vorticity form a shear layer. 
Approximately one chord length downstream, an instability forms (Kelvin-Helmholtz) resulting in a roll-up of the shear layer. As the flow progresses downstream, viscous diffusion causes the magnitude of vorticity to diminish as the structures advect downstream.

Figure 6 shows a Blasius boundary layer profile computed from a flat plate solution at chord stations of 0.7 and $0.9 x / c$. These calculations were conducted to validate the code and lend credibility to laminar boundary layer results. The computation shows reasonable agreement with the theoretical solution and do exhibit the self-similar profiles hypothesized. Slight differences in the profile may be attributed to the fact that a $1 \%$ thick flat plate with a cylindrical leading edge was used. Research conducted by Narasimha and Prasad [6] suggests a flat or cylindrical leading edge may cause slight flow separation near the leading edge altering the boundary layer profiles.

Figure 7 shows the boundary layer profiles over the NACA 0012 airfoil at $0^{\circ}$ angle of attack. Results seem reasonable compared with the flat plate solutions. Fuller velocity profiles are seen closer to the leading edge $(x / c=0.15)$. Effect of the adverse pressure gradient results in a flatter profile by $x / c=0.75$.

Colored plots of the vorticity distribution and streaklines around a NACA 0012 airfoil at $15^{\circ}$ angle of attack and $t=7.0$ are shown in Fig. 8. The streaklines were constructed using FIELDVIEW visualization software. In order to compute the streaklines, velocity values were computed on a fixed structured grid fitted to the body. The streaklines were computed as particle path trajectories. While there will exist difference between the streaklines and streamlines, this technique allows for visualization of the coherent boundary layer structures. As can be seen in the vorticity distribution, concentrations of both red (positive) vorticity as well as blue (negative) vorticity can be seen in the separated region near the trailing edge. Streakline plots show a coherent vortex pair being shed off the trailing edge as well as cohesive boundary layer vortices at mid-chord with a clockwise sense of rotation.

Development of the separated region over the NACA 0012 airfoil at $15^{\circ}$ angle of attack was then examined in more detail. Examination of the surface vorticity values provided evidence of incipient separation. A plot of the surface vorticity over the upper surface downstream of midchord is shown in Fig. 9. Initially, the surface vorticity is negative over the upper surface. At $t=0.5$, the vorticity remains approximately constant at 60 from $x / c=0.4-0.8$. Afterward, the vorticity magnitude decreases and changes sign at approximately $x / c=0.95$ suggesting trailing edge separation. By $t=1.0$, the surface vorticity magnitude decreases over the aft portion of the airfoil and changes sign at $x / c=0.875$. By $t=1.5$, the vorticity changes $\operatorname{sign}$ at $x / c=0.6$ and by $t=3.0$, the vorticity changes sign by $x / c=0.45$.

The point where the vorticity changes sign was defined as the separation point. It was then possible to quantitatively examine the temporal development of the separated region. Figure 10 shows this point of separation as a function of time for all positive angles of attack examined. For the $5^{\circ}$ test case, the flow remains completely attached out to $t=2.0$. The separation point then moves upstream reaching a value of $x / c=0.8$ by $t=4.0$. Afterward, the separation point fluctuates somewhat but retains an average value of approximately 0.8 . By $\alpha=10^{\circ}$, flow separation is much more pronounced. The flow remains completely attached out to approximately $t=1.0$. Afterward, the separation point rapidly progresses upstream reaching $x / c=0.45$ by $t=6.0$. For $\alpha=15^{\circ}$ and $20^{\circ}$, the flow remains attached only briefly and the separation point moves upstream immediately after the flow was accelerated. The separation point reaches $x / c=0.35$ for the $15^{\circ}$ case and reaches $x / c=0.3$ for the $20^{\circ}$ case. In both cases, there does appear to be large variations in the separation point due to vortex shedding. Although not shown, visualization of the flow field revealed correlation between movement of the separation point downstream with shedding of boundary layer vortices into the wake.

Average values of the surface vorticity and surface pressure are plotted in Figs. 11 and 12 
respectively. Time average values were taken between $t=4.0$ and 7.0. As can be seen, the leading negative vorticity peaks are considerably sharper as angle of attack is increased. In addition, there is a sharp increase in positive vorticity at the trailing edge. For this airfoil, resolution of the trailing edge allowed for the computation of this strong positive vorticity. The resultant pressure distributions shown in Fig. 12 show that leading edge suction pressure peaks continue to persist even out to a $20^{\circ}$ angle of attack.

\section{Conclusions}

A novel numerical technique has been used to compute the unsteady flow development about a NACA 0012 airfoil at positive angles of attack. Vorticity is specified at a number of points (nodes) in the field. Velocities at the nodal points are determined according to the Biot-Savart integral. In order to perform the integration, triangulation of the vorticity field is accomplished. This has the advantage that the velocity at the nodal points will be finite. Triangulation of the vorticity field made it possible to compute derivatives using a least-squares formulation. This allowed for a deterministic solution of the diffusion term in the vorticity equation.

Initially, the vorticity is confined to the surface and is determined in order to satisfy the no-slip boundary conditions. Since the Laplacian is computed directly, it is possible to diffuse the surface vorticity into the flow. The vorticity is directly diffused to nodal points in the flow thus evolving the vorticity at these points. The nodes are then advected by the local flow and thus inherently adapt to continuously changing flow conditions. Distribution of vorticity in the surface and the flow then account for the boundary layer and wake.

In order to test the effectiveness of the current technique, flow past a NACA 0012 airfoil at angles of attack was examined. This challenging problem allows for investigation of the attached as well as separated flow. In fact, it was possible to examine incipient separation for flow past the airfoil at high angles of attack. At zero and five degrees angle of attack, reasonable prediction of the flow was accomplished. Boundary layer profiles on a flat plate agreed well with the Blasius boundary layer solution. Boundary layer profiles over the airfoil seemed reasonable in terms of both thickness and shape. The velocity profiles were fuller in the region of favorable pressure gradient and became flatter in the region of adverse pressure gradient.

Examination of flow separation revealed initial laminar separation at approximately $80 \%$ chord for the $5^{\circ}$ angle of attack test case. This is consistent with low Reynolds number for flow past symmetrical airfoils (see van Dyke [10]). As angle of attack was increased, the separation point moved up the airfoil as expected. The separation point, however, never reached the leading edge region. Even at $20^{\circ}$ angle of attack, the separation point never moved upstream of $30 \%$ chord. This result is not consistent with experimental data. At $20^{\circ}$ angle of attack, the flow should separate almost immediately from the leading edge due to the strong adverse pressure gradients. At least two explanations for this inconsistency are offered. First, the flow field may still be undergoing an unsteady development process. As the flow initially separates, cohesive vortex structures form which temporarily re-attach the flow. As these vortices are shed, subsequent vortices are weaker due to less vorticity being produced and eventually, separation moves closer to the leading edge. Another possibility for this inconsistency is associated with using the log of the vorticity instead of the vorticity itself to evolve the vorticity field. While this method has much better numerical stability characteristics, it does require vorticity of each sign to be treated separately. This likely becomes important during boundary layer separation where both signs of vorticity are produced. A modified scheme may be required to treat the case where vorticity of both signs must be diffused together. 
Previous work utilized this method to compute unsteady flow past a cylinder (Huyer and Grant [4]). These results were in excellent agreement with experimental data and demonstrated the effectiveness of the method to compute bluff body separation. This same method has also been used to compute attached boundary layers with considerable accuracy. In both cases, fewer than 10000 points were required to compute the boundary layer and wake of the airfoil and circular cylinder. As shown in this paper, the method has the potential to predict flow separation past streamlined bodies. Future work will examine the separation problem in more detail. This especially includes unsteady flow separation past maneuvering bodies. Finally, this approach is being extended to compute three-dimensional unsteady flow (Grant et al. [1]).

\section{Acknowledgements}

This research was supported by the Office of Naval Research, Contract No. 95WX20287, Mr. James A. Fein, Program Manager and by the NUWC Internal Research program, Dr. Stuart Dickinson, program manager.

\section{References}

[1] Grant, J.R., Huyer, S.A. and Uhlman, J.S., "Solution of the Vorticity Equation on a Lagrangian Mesh Using Triangularization: Computation of the Biot-Savart Integral in Three-Dimensions," Proc. Forum on Vortex Methods for Eng'n Appl., Albuquerque, NM, February, 1995.

[2] Greengard, L. and Rokhlin, V., "A Fast Algorithm for Particle Simulations," Math. Comp., 47, pp. 387-398, 1987.

[3] Huyer, S.A., Grant, J.R. and Uhlman, J.S., "Computation of Unsteady Separated Flow Fields Past an Oscillating Airfoil Using Discrete Vortex Elements," AIAA Paper 94-2257, Colorado Springs, CO, 1994.

[4] Huyer, S.A. and Grant, J.R., "Incorporation of Boundaries for 2D Triangular Vorticity Element Methods," Proc. Forum on Vortex Methods for Eng'n Appl., Albuquerque, NM, February, 1995.

[5] Kempka, S.N. and Strickland, J.H., "A Method to Simulate Viscous Diffusion of Vorticity by Convective Transport of Vortices at a Non-Solenoidal Velocity," Sandia National Laboratories Report SAND93-1763, August, 1993.

[6] Narasimha, R. and Prasad, S.N., "Leading Edge Shape for Flat Plate Boundary Layer Studies," Fluid Mechanics Rep. 84 FM 3, Dept. Aerospace Engineering, Indian Institute of Science, 1984.

[7] Peraire, J., Morgan, K. and Peiro, J., "Numerical Grid Generation," von Karman Institute for Fluid Dynamics Lecture Series 1990-06, Unstructured Mesh Methods for CFD, N90-29611, June, 1990.

[8] Russo, G. and Strain, J.A., "Fast Triangulated Vortex Methods for the 2D Euler Equations," J. Comp. Phys., 111, pp. 291-323, 1994.

[9] Uhlman, J.S. and Grant, J.R., "A New Method for the Implementation of Boundary Conditions in the Discrete Vortex Element Method," ASME 1993 Fluids Engineering Spring Meeting, Washington, DC, June, 1993. 
[10] van Dyke, M., An Album of Fluid Motion, The Parabolic Press, Stanford, CA, p. 25, 1982.

[11] Zienkiewicz, O.C., The Finite Element Method, 3rd edition, McGraw Hill Publishing Co., pp. 165-169, 1977.

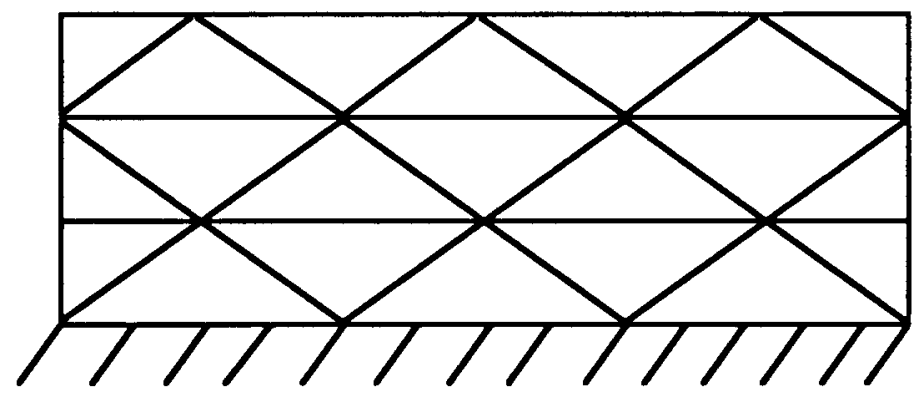

Figure 1: Initial surface nodal distribution.

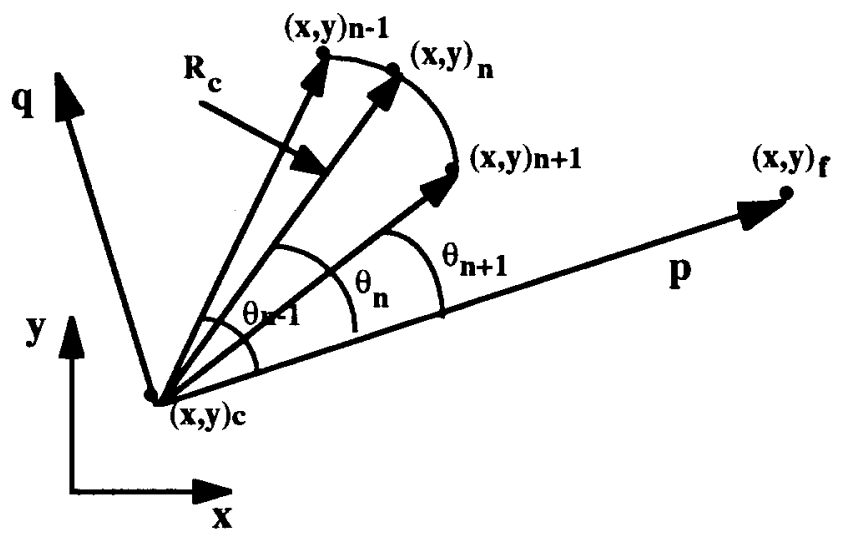

Figure 2: Surface source panel geometry. 


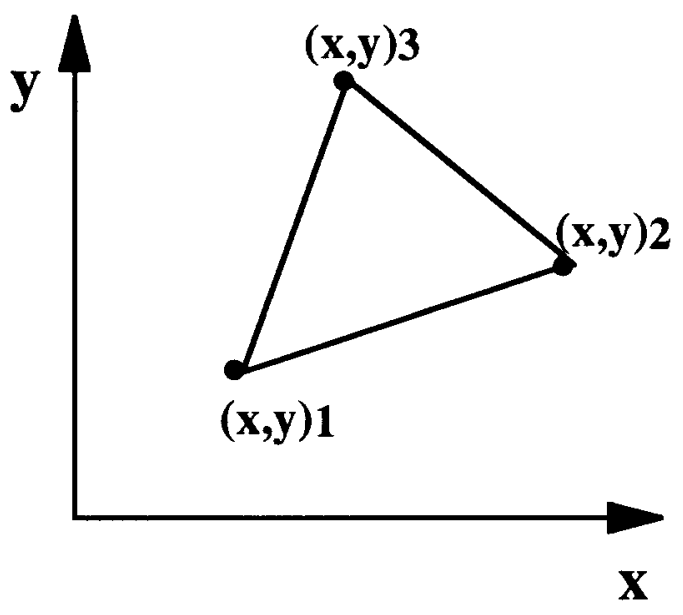

Figure 3: Triangular vorticity element geometry.

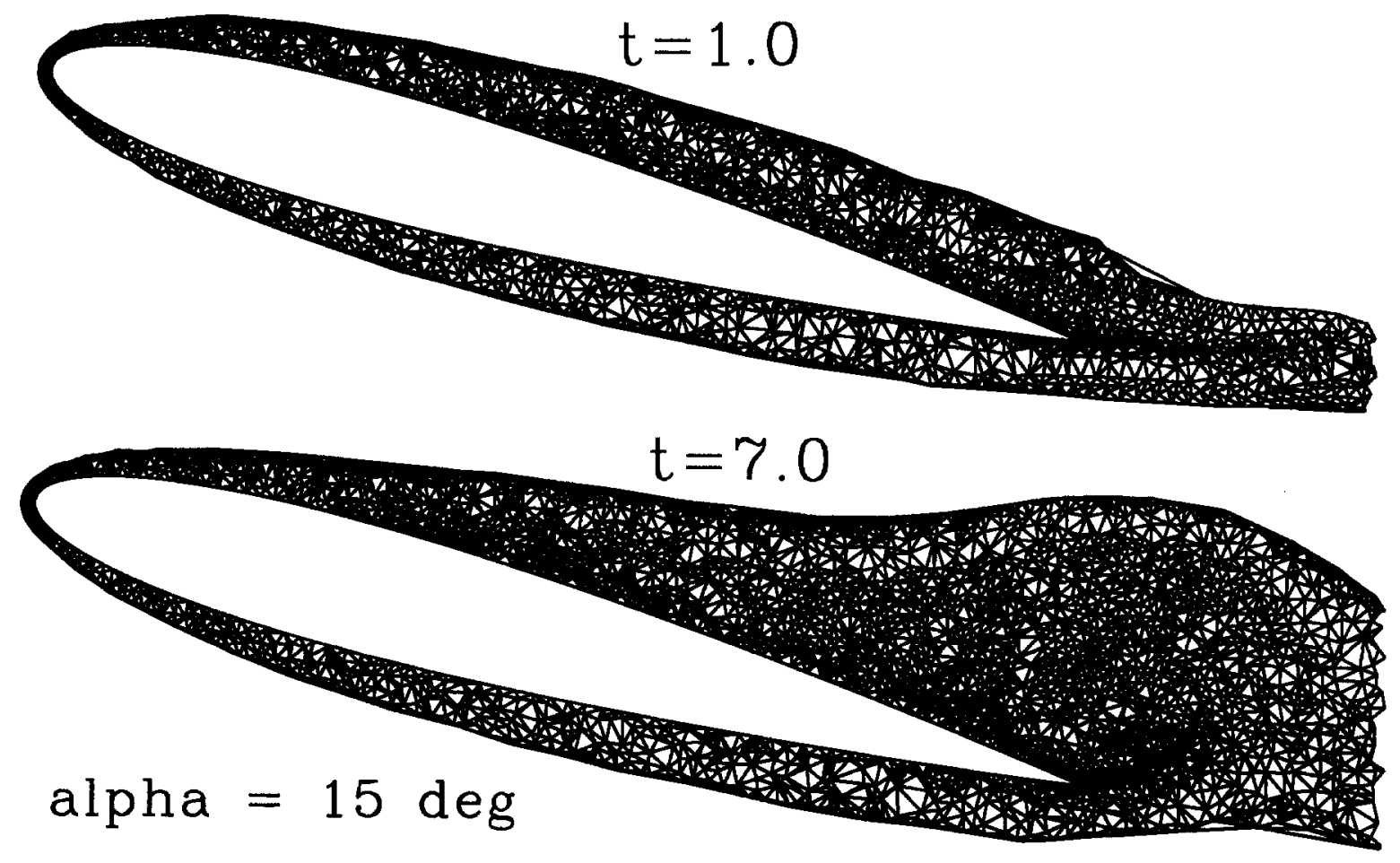

Figure 4: Triangular meshes over a NACA 0012 airfoil for $\alpha=15^{\circ}, R e=5000$ at $t=1.0$ and 7.0. Mesh downstream of $x / c=1.25$ is not shown. 


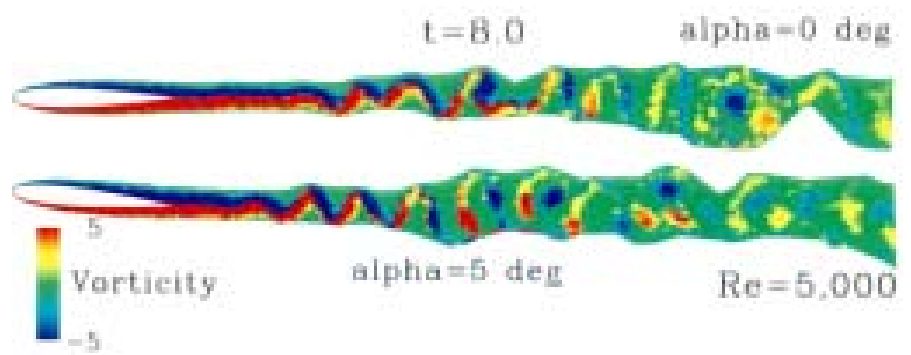

Figure 5: Vorticity plots showing unsteady wake development past a NACA 0012 airfoil at $\alpha=0^{\circ}$ and $5^{\circ}$ at $t=8.0$.

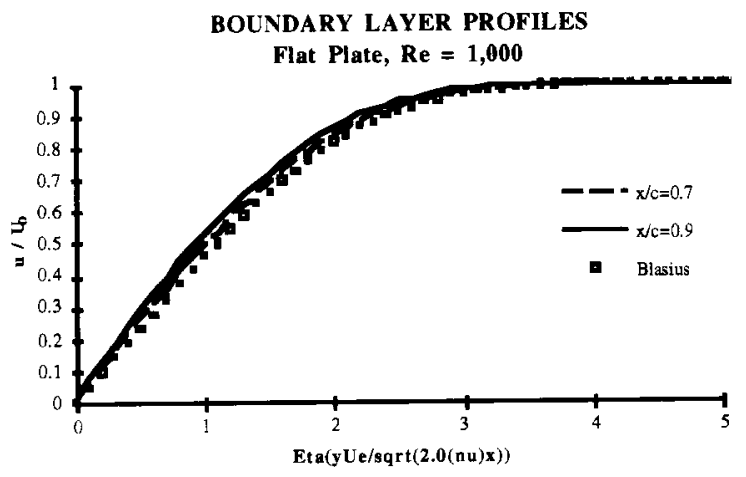

Figure 6: Flat plate boundary layer velocity profiles for $R e=1000$ at $x / c=0.7$ and 0.9 compared with Blasius profile.

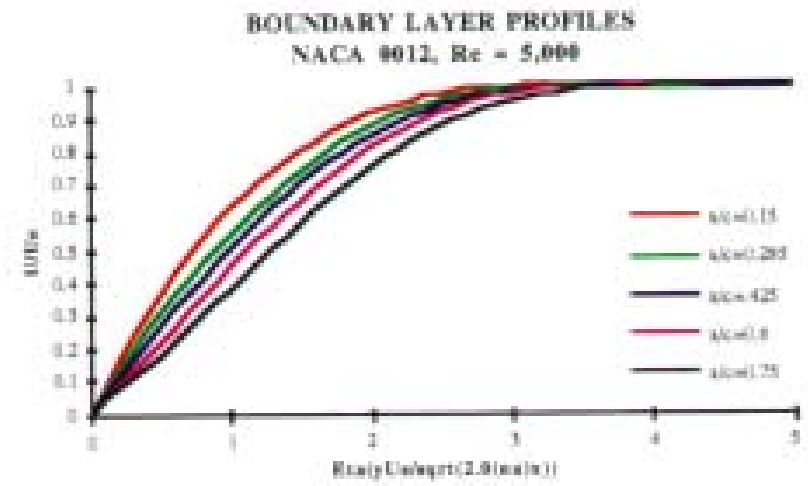

Figure 7: NACA 0012 boundary layer velocity profiles for $R e=5000$ from $x / c=0.15$ to 0.75 . 

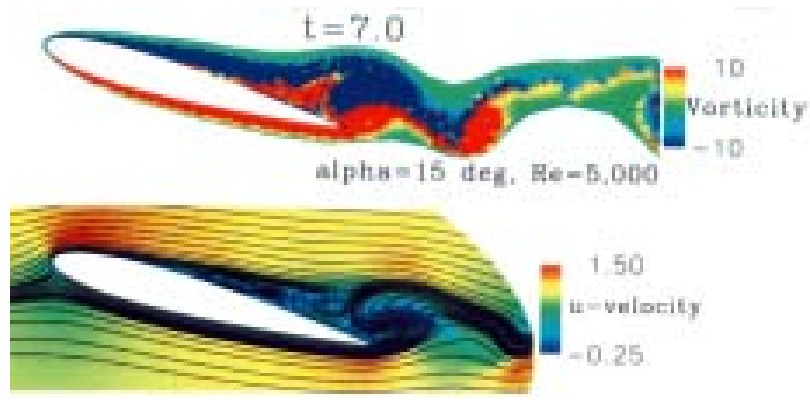

Figure 8: Vorticity distributions and flow streaklines past a NACA 0012 airfoil for $R e=5000$ and $\alpha=5^{\circ}$ at $t=7.0$.

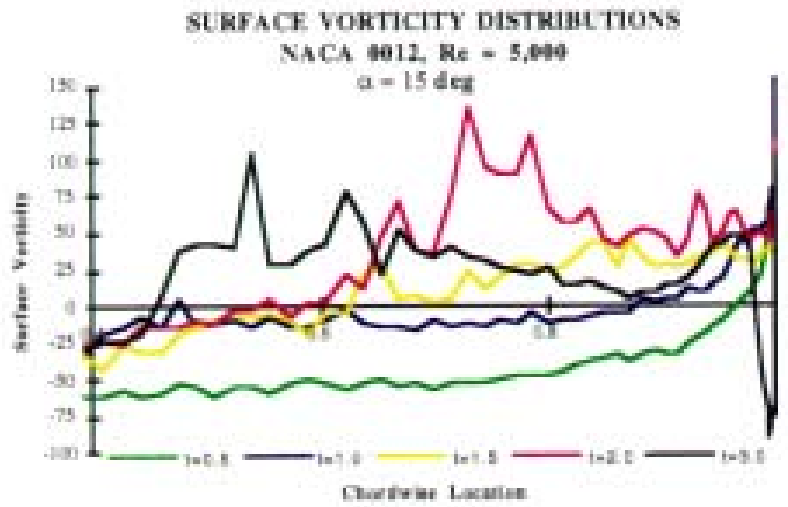

Figure 9: Surface vorticity distributions for $R e=5000$ and $\alpha=5^{\circ}$ from $t=0.5$ to 3.0.

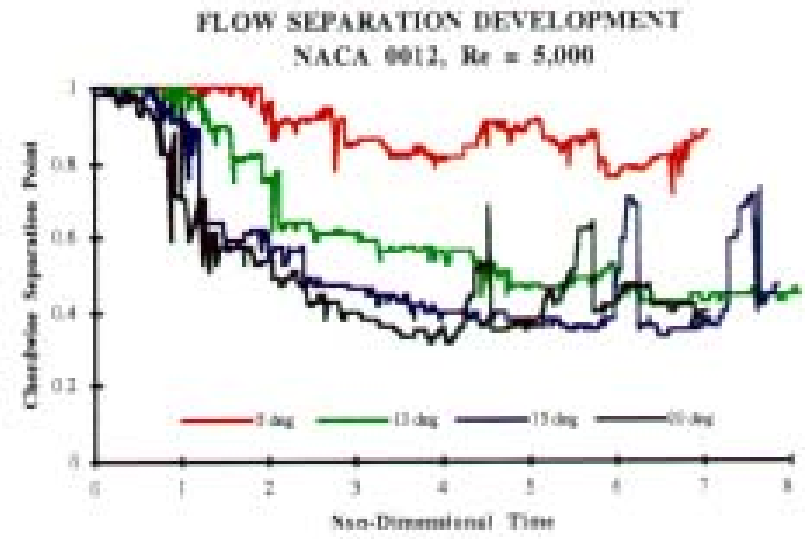

Figure 10: Chordwise separation as a function of time for $\alpha=5^{\circ}, 10^{\circ}, 15^{\circ}$ and $20^{\circ}$. 


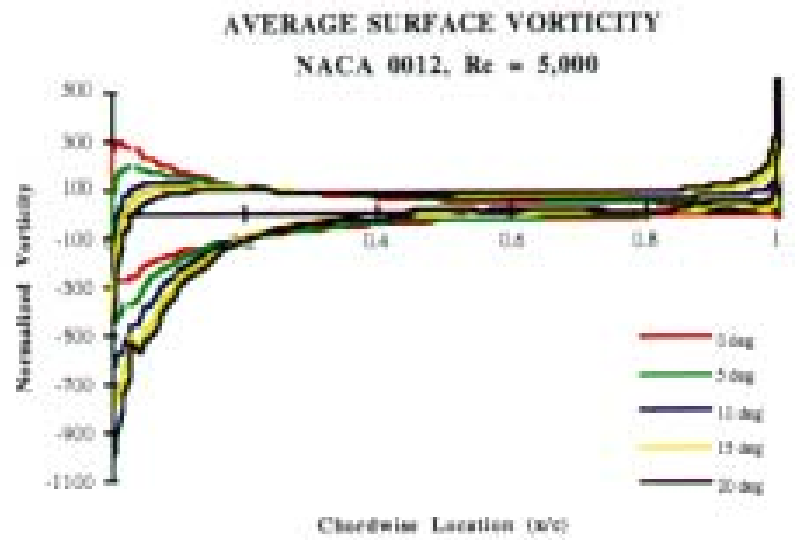

Figure 11: Time averaged $(t=4.0-7.0)$ surface vorticity distributions for $\alpha=0^{\circ}, 5^{\circ}, 10^{\circ}, 15^{\circ}$ and $20^{\circ}$.

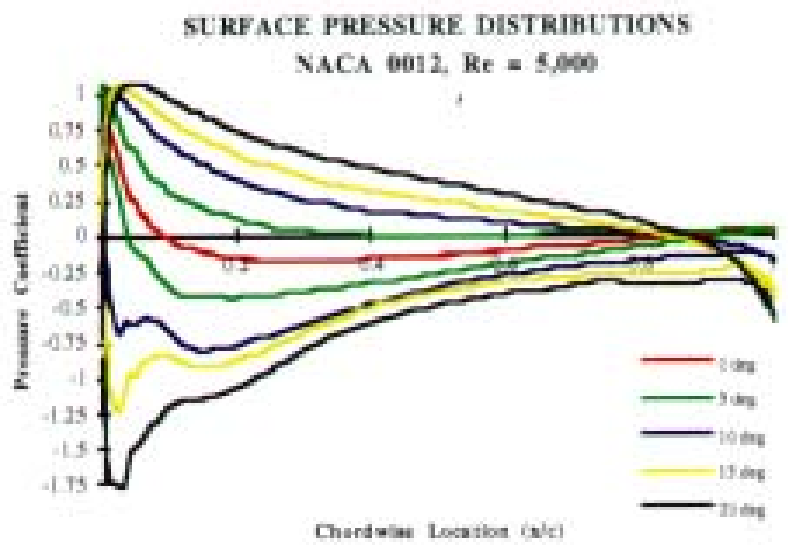

Figure 12: Time averaged $(t=4.0-7.0)$ surface pressure distributions for $\alpha=0^{\circ}, 5^{\circ}, 10^{\circ}, 15^{\circ}$ and $20^{\circ}$. 